\title{
LATTICE BOLTZMANN SIMULATION OF A SINGLE DROPLET IMPINGEMENT AND EVAPORATION ON INCLINED HEATED SURFACE
}

\author{
RUI MA ${ }^{1}$, JING GONG $^{1}$, YONG WANG ${ }^{2}$, WEIZHONG LI $^{2} \&$ BO DONG \\ ${ }^{1}$ College of Mechanical and Transportation Engineering, China University of Petroleum, Beijing, China. \\ ${ }^{2}$ Key Laboratory of Ocean Energy Utilization and Energy Conservation of Ministry of Education, \\ Dalian University of Technology, Dalian, P. R. China.
}

\begin{abstract}
The impingement and evaporation processes of droplet widely exist in many industrial fields such as fuel injection in combustion engines, spray drying and turbines. When a single droplet falls and impacts on an inclined hot surface under the effect of gravity, it evaporates after contacting with the surface due to the heated transfer. The inclined angle of surfaces has great effects on droplet dynamics and heat transfer. In this work, the pseudo-potential model and a thermal lattice Boltzmann model are combined to simulate the impact process and the heat transfer. Moreover, the Peng-Robinson equation of state is incorporated in the effective density function to consider the large liquid/gas density ratio. The influences of inclined angle on droplet shape and evaporation rate are obtained and analyzed. The results show that for a fixed initial velocity, when the inclined angel increases, droplet deformation is significant as the motion between droplet and the surface is strengthened and the droplet evaporation rate gets faster since the heat transfer is enhanced.

Keywords: droplet evaporation, impingement, inclined surface, non-ideal equation of state, pseudopotential model
\end{abstract}

\section{INTRODUCTION}

The flow of liquids over heated solid surfaces occurs in a variety of engineering and technological applications, such as falling-film evaporators, spray drying, fuel injection in combustion engines, and gas turbines. In all the aforementioned applications, the dynamic behavior of the impinging droplets and the heat transfer between the droplets and the hot surfaces are important factors, which affect the mass transfer associated with liquid-vapour phase change. The fluid flow associated with droplet impingement is rather complicated and not understood in detail. Different characteristic features are observed on the basis of special circumstances [1]. For example, when raindrops falling into a pool it can splash, coalesce with or bounce off the water. If the impact energy is high enough, the impact of a droplet on a liquid surface leads to the formation of a crater in the liquid. Therefore, research on droplets impacting on a solid surface attracts great interest from researchers. Consequently, the solid/ liquid/gas interfacial phenomena need to be studied, and some researchers have carried out experimental as well as numerical studies.

In the experiment field, for instance, Crafton and Black [2] experimentally observed the evaporation rates of small liquid droplets and measured heat transfer rates when individual droplets evaporated on a horizontal heated surface. The results show that the trends in the wetted diameter, height and contact angle for water droplets are fundamentally different from the geometric changes for heptane droplets. Bhardwajet et al. [3] investigated the coupling of fluid dynamics, heat transfer and mass transfer during the impact and evaporation of droplets on a heated solid substrate. The results showed that the interfacial temperature first increases exponentially and then decreases linearly. 
Apart from the above-mentioned experimental researches, there are also many numerical studies to simulate the impact of droplet dynamics on the heated surface. And these numerical studies can be divided into two categories: the Navier-Stokes equation-based solvers and the lattice Boltzmann method. Alternate to conventional Navier-Stokes equation-based solvers, the lattice Boltzmann method has been successfully used to perform efficient computations of multiphase flow problems. The first model is the so-called Rothman-Keller LB model using color gradients to separate and model the interaction at the multiphase interfaces, which was proposed by Gunstensen et al. [4] in 1991. The second one is the pseudo-potential model proposed by Shan and Chen [5] in which a pseudo-potential is introduced to account for the nonlocal particle interactions. Another model is the free-energy model proposed by Swift et al. [6] in which phase effects are directly introduced into the collision process by considering a generalized equilibrium distribution function that includes a non-ideal pressure tensor term. There are also other LB multiphase models such as the one proposed by He et al. [7] in which a mean-field method was developed where a proper force term was derived for multiphase behavior.

Among all the methods mentioned before, the pseudo-potential model is one of the most widely used LB multiphase model due to its simplicity and versatility. The basic idea is to represent the microscopic molecular interactions at the mesoscopic scale using a pseudopotential depending on the local density. With such interactions, a single component single fluid spontaneously segregates into two-phases with high and low densities when the interaction strength is under the critical point. Such automatic phase separation is an attractive characteristic of the pseudo-potential model, as the phase interface is no longer a mathematical boundary and no explicit interface-tracking or interface-capturing technique is needed. The densities change smoothly from one bulk value to another across the phase interface which usually has the width of several lattice nodes. Due to its remarkable computational efficiency and clear representation of the underlying microscopic physics, this model has been a promising technique for simulating and investigating multiphase flow problems [8].

Since the mechanism of deformation and breakup of liquid droplet impingement on an oblique plane is the research point of this paper. The process of the impact of a liquid droplet on a heated flat solid surface was simulated when considering the interfacial forces of solidliquid and liquid-gas. The overall goal is to show the droplet shape and to analyze the effect of the inclined angle on the droplet deformation and evaporation process.

\section{DESCRIPTION OF THE SIMULATION MODEL}

Since we need a multiphase LBM model and a corresponding energy equation model to simulate the phase change process, two particle distribution functions (the density distribution function and the temperature distribution function) are required. These two functions are coupled through the temperature term. In this section, we will give a brief introduction to the relevant equations and the source term which is related to phase-change.

\subsection{The multiphase equation}

A two-dimensional nine-velocity pseudo-potential LBM was used. The discrete Boltzmann equation with the BGK form of collision operator is given by

$$
f_{i}\left(\boldsymbol{x}+\boldsymbol{e}_{i} \Delta t, t+\Delta t\right)=f_{i}(\boldsymbol{x}, t)-\frac{1}{\tau_{f}}\left[f_{i}(\boldsymbol{x}, t)-f_{i}^{e q}(\boldsymbol{x}, t)\right]+\Delta f_{i}
$$


where $f_{i}$ is the density distribution function, $t$ is time and $\Delta t$ is the time step. The lattice velocity is given by $\boldsymbol{e}_{i}, \boldsymbol{x}$ is the particle position, and $\tau_{f}$ is the relaxation times. The body force is expressed by $\Delta f_{\alpha}$. Since the D2Q9 lattice is used to conduct the simulations, the discrete velocity vectors are given as follows:

$$
\boldsymbol{e}_{i}=\left\{\begin{array}{c}
(0,0), i=0 \\
( \pm 1,0),(0, \pm 1) c, i=1,2,3,4 \\
( \pm 1, \pm 1) c, i=5,6,7,8
\end{array}\right.
$$

where $c=\Delta x / \Delta t$ is the lattice speed, with $\Delta x$ being the lattice spacing and $\Delta t$ being the time spacing. Note that $c_{s}^{2}=c^{2} / 3$ in D2Q9 scheme. The kinematic viscosity is given by

$$
v=c_{s}^{2}\left(\tau_{f}-0.5\right) \Delta t
$$

The equilibrium distribution functions $f_{\alpha}^{e q}$ is calculated by:

$$
f_{i}^{e q}=\omega_{i} \rho\left[1+\frac{\boldsymbol{e}_{i} \cdot \boldsymbol{u}}{c_{s}^{2}}+\frac{\left(\boldsymbol{e}_{i} \cdot \boldsymbol{u}\right)^{2}}{2 c_{s}^{4}}-\frac{\boldsymbol{u}^{2}}{2 c_{s}^{2}}\right]
$$

here the weighting factor $\omega_{i}$ are defined as $\omega_{0}=4 / 9, \omega_{1-4}=1 / 9, \omega_{5-8}=1 / 36$. The density and velocity in the above mentioned equations is obtained by:

$$
\begin{gathered}
\rho=\sum_{i} f_{i} \\
\rho \boldsymbol{u}=\sum_{i} f_{i} \boldsymbol{e}_{i} .
\end{gathered}
$$

Note that $\boldsymbol{u}$ in eqn. (6) is not the real fluid velocity. The real fluid velocity $\mathbf{U}$ is given by

$$
\boldsymbol{U}=\boldsymbol{u}+\frac{\Delta t \boldsymbol{F}}{2 \rho}
$$

There are many methods to incorporate the force term in LBM. In this paper, the exact different method is adopted, which has been demonstrated to have better accuracy and more appropriate for real gases. The force term $\Delta f_{\alpha}$ in eqn. (1) is defined as [9]:

$$
\Delta f_{i}=\omega_{i} \delta_{t}\left[\frac{\boldsymbol{e}_{i} \cdot \boldsymbol{F}}{c_{s}^{2}}+\frac{\left(\boldsymbol{e}_{i} \cdot \boldsymbol{U}\right)\left(\boldsymbol{e}_{i} \cdot \boldsymbol{F}\right)}{c_{s}^{4}}-\frac{\boldsymbol{U} \cdot \boldsymbol{F}}{c_{s}^{2}}\right]
$$

here, $\boldsymbol{F}$ is the external force, which include the interparticle interaction force $\boldsymbol{F}_{\text {int }}$, gravitational force $\boldsymbol{F}_{g}$ and the interaction force $\boldsymbol{F}_{s}$ between solid surface and fluid.

$$
\boldsymbol{F}=\boldsymbol{F}_{i n t}+\boldsymbol{F}_{g}+\boldsymbol{F}_{s}
$$

Then the interparticle interaction force $\boldsymbol{F}_{\text {int }}$ can be calculated by [10]:

$$
F_{\mathrm{int}}=-\beta \Phi(x) \sum_{i=1}^{8} \lambda_{i} \Phi\left(x+e_{i}\right) e_{i}-\frac{1-\beta}{2} \sum_{i=1}^{8} \lambda_{i} \Phi^{2}\left(x+e_{i}\right) e_{i} .
$$


For the Peng-Robinson equation of state (P-R EOS), a value of $\beta=1.16$ has been shown to yield an accurate reproduction of the coexistence curves in comparison to pure local or pure mean value approximations. In order to incorporate various EOS into the pseudo-potential model, a parameter is defined to describe the interaction potential which depends on equation of state:

$$
f=\sqrt{\frac{2\left(p-\rho c_{s}^{2}\right)}{c_{0} g}}
$$

here, $c_{0}=6$ and $g$ is the interaction strength. The P-R EOS is suitable to investigate hydrocarbon droplets evaporating in a high-pressure and high-temperature environment and expressed as:

$$
p=\frac{\rho R T}{1-b \rho}-\frac{a \rho^{2} \alpha^{\prime}(T)}{1+2 b \rho-b^{2} \rho^{2}}
$$

with $a=0.45724 R^{2} T_{c}^{2} / p_{c}$ and $b=0.0778 R T_{c} / p_{c}$. The parameter $\alpha^{\prime}(T)=[1+(0.37464+$ $\left.\left.1.54266 \omega-0.26992 \omega^{2}\right) \times\left(1-\sqrt{T / T_{c}}\right)\right]^{2}$, here $\omega$ is the acentric factor of the fluid.

The gravitational force $\boldsymbol{F}_{g}$ is calculated by

$$
\boldsymbol{F}_{g}=\left(\rho(\boldsymbol{x})-\rho_{\text {eva }}\right) \boldsymbol{g}
$$

where $\boldsymbol{g}$ is the gravitational acceleration vector and $\rho_{\text {eva }}$ the average density of the entire domain.

The interaction force $\boldsymbol{F}_{s}$ between solid surface and fluid can be defined as

$$
\boldsymbol{F}_{s}=-G_{s} \rho(\boldsymbol{x}) \sum_{i} \omega_{i} s\left(\boldsymbol{x}+\boldsymbol{e}_{i} \Delta t\right) \boldsymbol{e}_{i}
$$

here, $G_{s}$ is the parameter denoting the fluid-solid interaction strength which can be implemented to control wetting conditions on solid surface. Once the force term $\boldsymbol{F}$ is obtained, the macroscopic velocity is updated by averaging the momentum before and after the collision.

\subsection{The thermal equation}

In order to simulate phase change problem in multiphase flow, the multiphase equation $f_{i}$ is coupled with another distribution function $g_{i}$ to ensure the conservation of energy. The D2Q9 scheme is also employed in the energy distribution function to compute the temperature field. The discrete Boltzmann equation for temperature is given by:

$$
g_{i}\left(\boldsymbol{x}+\boldsymbol{e}_{i} \Delta t, t+\Delta t\right)=g_{i}(\boldsymbol{x}, t)-\frac{1}{\tau_{g}}\left[g_{i}(\boldsymbol{x}, t)-g_{i}^{e q}(\boldsymbol{x}, t)\right]+G_{i}
$$

where $\tau_{g}$ is the relaxation time. The corresponding equilibrium distribution is determined as:

$$
g_{\alpha}^{e q}=\omega_{i} \rho T\left(1+\frac{\boldsymbol{e}_{\alpha} \cdot \boldsymbol{U}}{c_{s}^{2}}\right)
$$

The temperature $T$ can be obtained by:

$$
T=\sum_{i} g_{i}
$$


The parameter $G_{i}$ in eqn (15) is the correction term derived from the phase change process, which is proposed as [11]:

$$
G_{i}=\omega_{i}\left[\frac{\nabla \cdot(\kappa \nabla T)}{c_{p}}-\nabla \cdot[\alpha \nabla(\rho T)]\right]
$$

where $\kappa$ is thermal conductivity, $c_{p}$ is the specific heat, and the thermal diffusivity is given as

$$
=c_{s}^{2}\left(\tau_{g}-0.5\right) \Delta t
$$

By implementing the Chapman-Enskog expansion, the macroscopic energy equation for multiphase flows is recovered as:

$$
\frac{\partial T}{\partial t}+U \cdot \nabla T=\frac{\nabla \cdot(\kappa \nabla T)}{\rho c_{p}}+\phi .
$$

\section{MODEL VALIDATION}

In this section, the Maxwell construction for non-ideal EOS was introduced to obtain the corresponding coexistence curves. The coexistence curve of P-R EOS was investigated in order to validate the proposed model.

Initially, the density was set slightly higher than the critical density $\rho_{c}$ at the central domain and lower than $\rho_{c}$ elsewhere under a given temperature $T$. The droplet was placed in a $100 \times 100$ lattice region and the boundary conditions were set to be periodic on all directions. In the simulation, the relaxation times were set as $\tau_{f}=0.52$ and $\tau_{g}=0.53$, respectively. When a steady state was reached, the densities of droplet and vapour were compared with the analytical solutions given by Maxwell construction. As it was shown in Fig. 1, simulation results of the densities agreed well with analytical solutions both in the liquid branch $\left(\rho_{r}>1\right)$ and vapour branch $\left(\rho_{r}<1\right)$. Here, $T_{r}, \rho_{r}$ represent the reduced temperature and reduced density, respectively. When $T_{r}$ equals to $1.0, \rho_{r}$ has a unique value of 1.0 , which means that the

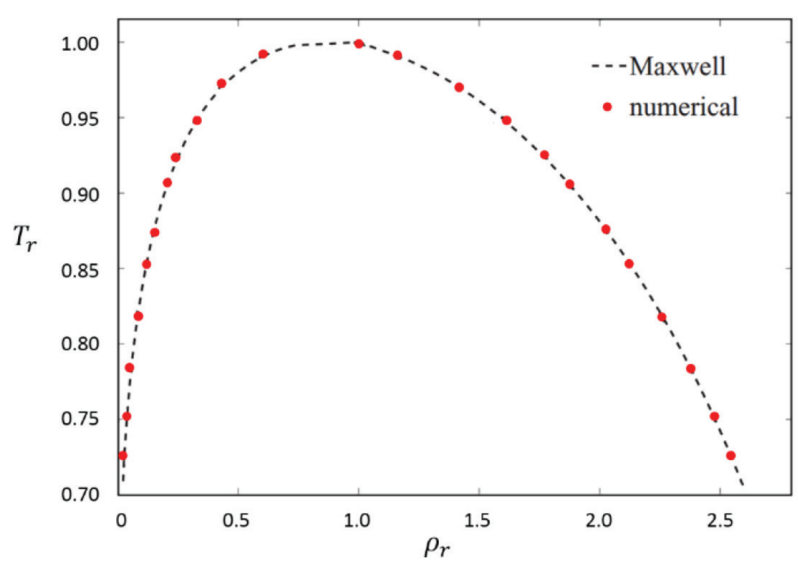

Figure 1: Reduced temperature $T_{r}$ as a function of the reduced density $\rho_{r}$.(P-R EOS with $\omega=0.30075$ ) 


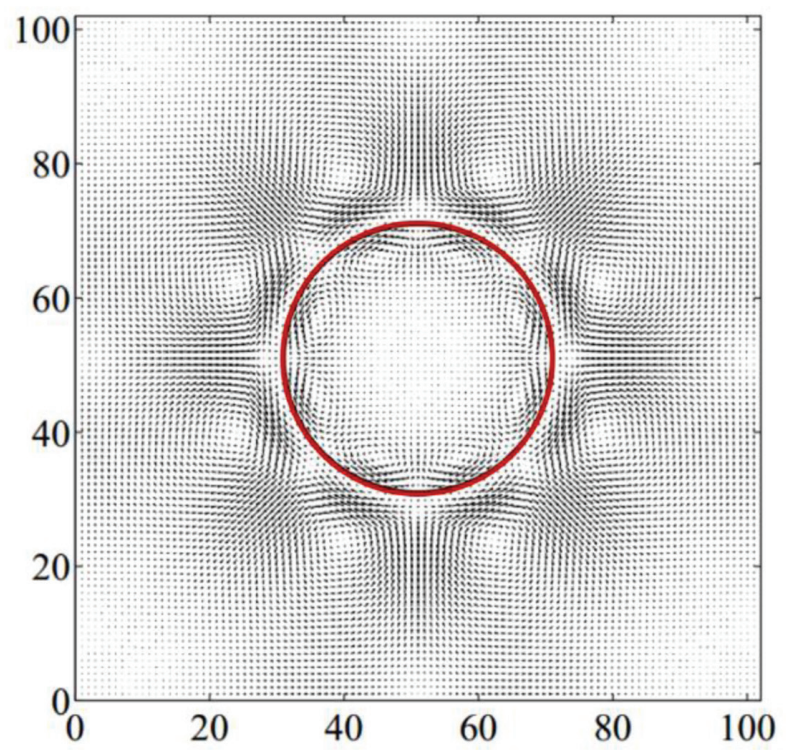

Figure 2: The velocity field of a static droplet at equilibrium condition $\left(T_{r}=0.85\right)$.

liquid is at the critical state. All variables in this paper are in lattice units, which can be converted into real physical properties based on the principal of reduced properties.

The velocity field for a typical equilibrium condition was demonstrated in Fig. 2. The profile of droplet was described by the red curve. The unphysical spurious currents are present and the maximum value is around the interface. This phenomenon naturally occurs for using a pseudo-potential model. The maximum spurious velocity observed in the equilibrium condition is $\left|v_{\max }\right| \sim 10^{-4}$, which is much smaller than the flow velocity which has the magnitude of $10^{-2}$ in the dynamic case when evaporation appears.

\section{LBM SIMULATION}

The inclined angle of surfaces has great effects on droplet dynamics and heat transfer. A 2D problem of a single droplet falls and impacts on an inclined hot surface when the effect of gravity was considered.

As shown in Fig. 3, a single droplet was placed in a gravitational field and an inclined plane which was located below the droplet. In this paper, three inclined angles, i.e. $15^{\circ} 30^{\circ}$ and $45^{\circ}$

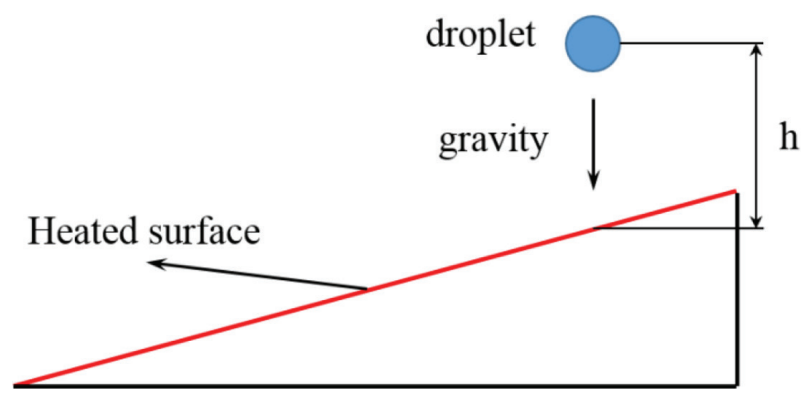

Figure 3: Physical situation of simulation 
were considered. The static droplet would move downward under the effect of gravity. For a certain time, the moving droplet will impact on the heated surface. Then the droplet started to experience deformation and evaporation.

\subsection{Deformation of droplet}

First, the system is set for the isothermal case and equilibrium conditions are achieved. Then, the effect of gravity and temperature distribution were added into the system. The droplet was accelerated under the action of gravity force. When the liquid droplet impingement on the heated inclined plane evaporation began because of the temperature imparity.

The non-equilibrium extrapolation scheme was adopted for a constant temperature boundary condition on the inclined plane. And a constant temperature boundary was set as $T_{w}=0.8 T_{r}$ on the hot inclined plane. The other three boundaries were considered to be full developed boundaries. The initial temperature of the saturated steam was defined as $T_{s}=0.75 T_{r}$. The value of fluid-solid interaction strength $G_{s}$ was chosen as 0.24 with corresponding contact angle of $120^{\circ}$. In the simulation, most parameters have the same value except the angles of the inclined plane. The relevant parameters were listed in Table 1.

The profiles of droplet at different time steps were selected to describe the deformation of droplet versus time. When the time step is smaller or equal to 10000 , the profile of droplet was selected once every 2000 time steps. For time step is greater than 10,000, the profile of droplet was selected once every 10,000 time steps. Figure 4 shows the shape evolution of a droplet with different inclination angles.

In all the three cases, distinctly, the volume of droplet decreased after it contacted with the inclined plane. Thermal energy was transferred from the heated plane to droplet because of temperature gradient. These transferred energies would lead to evaporation and the volume reduction occurred naturally. As shown in Fig. 4a, the shape of droplet was compressed by the effect of impingement. The droplet spread along the hot wall, and then it recovered to an ellipse-like shape. During this time, the impact effect was weaken and the surface tension would assemble the spread liquid film. This gather effect made the impacted droplet gathered into elliptical shape. When the angle of inclined plane increases gradually, as shown in Figs. $4 \mathrm{~b}$ and c, the droplets deformation was similar to the situation of Fig. 4a. The droplet was spread firstly and then assembled. It was worth noting that the droplet slip velocity (velocity slide along the inclined plane) was significantly different. The droplet on inclined plane with a larger angle had a greater slip velocity for a longer slip distance.

\subsection{Evaporation of droplet}

The mass decreasing of the impacted droplets versus time was expressed in Fig. 5. The mass of droplet was normalized by initial droplet mass. The value of 1.0 normalized mass means the mass quantity to initial droplet. The reduction of normalized droplet mass could be

Table1: Physical properties of liquid and vapour phases (P-R EOS with $\omega=0.30075$ ).

\begin{tabular}{llllllllll}
\hline Property & $\rho_{l} / \rho_{v}$ & $\alpha_{l}$ & $\alpha_{v}$ & $c_{p}^{l}$ & $c_{p}^{v}$ & $\rho_{l} / \rho_{c}$ & $\rho_{v} / \rho_{c}$ & $K_{l} / \rho_{c}$ & $K_{v} / \rho_{c}$ \\
Value & 42.07 & $1 / 30$ & $1 / 6$ & 2.62 & 2.50 & 2.60 & 0.0618 & 0.277 & 0.0258 \\
\hline
\end{tabular}



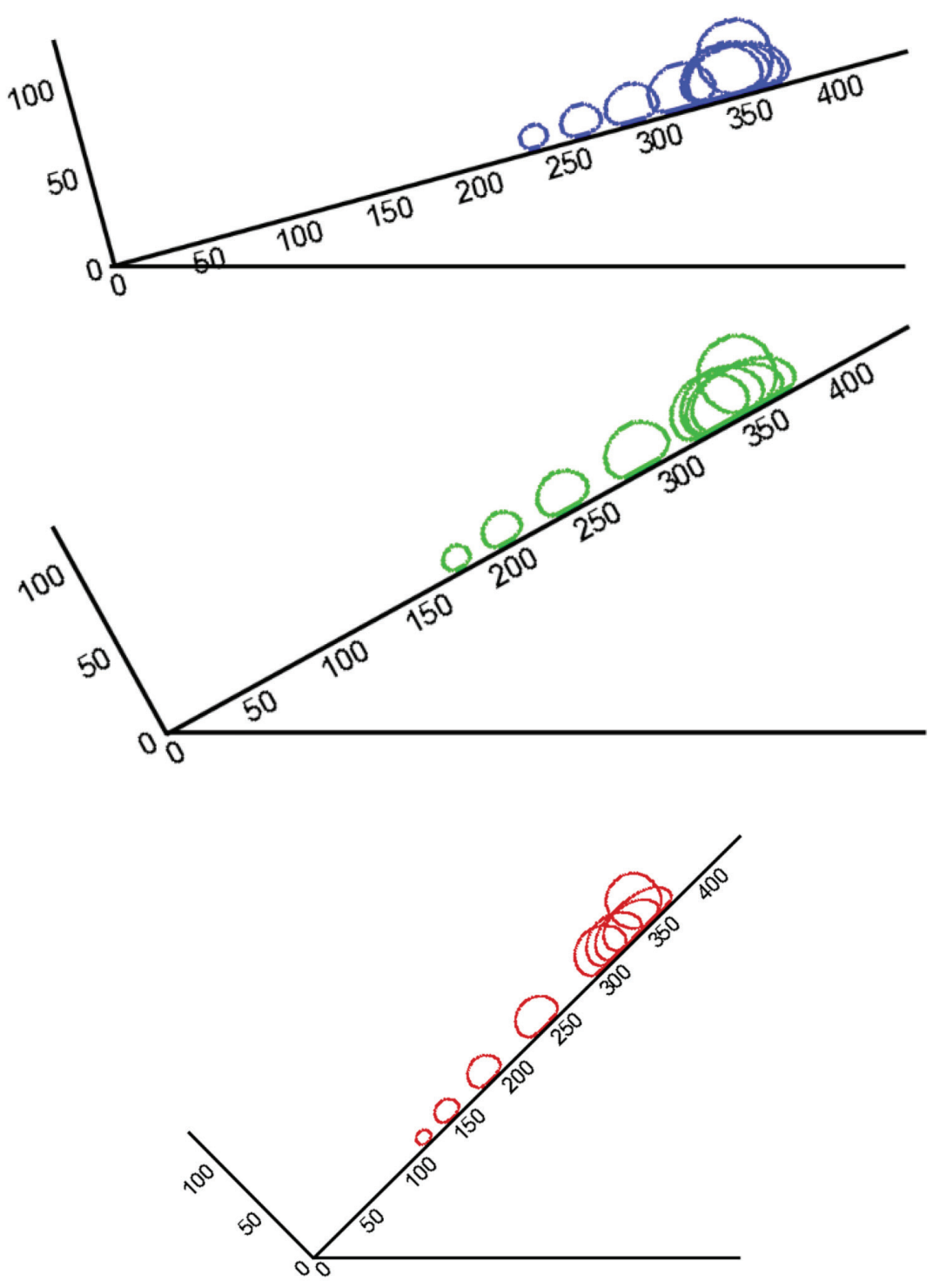

Figure 4: Shape evolution of a droplet for various inclination angles. (a) (inclined angle $=15^{\circ}$ ), (b) (inclined angel $=30^{\circ}$ ), (c) (inclined angle $=45^{\circ}$ ).

representing evaporation of droplets. On the whole, the mass of the droplet were decreasing as time goes on in all three cases. And what's more, as shown in Fig. 5, when the angles of inclined plane were $15^{\circ}$ and $30^{\circ}$, the decreasing mass of droplets were almost identical at the same time. However, the reduction of mass got more quickly when the angle of inclined plane was $45^{\circ}$. The relative motion between droplet and inclined plane could intensify heat transfer. Figure 6 shows the slip velocity of droplets versus time at different inclination angles. Obviously, the droplet on the larger angle of inclined plane had a higher slip velocity which could enhance the evaporation of droplet. 


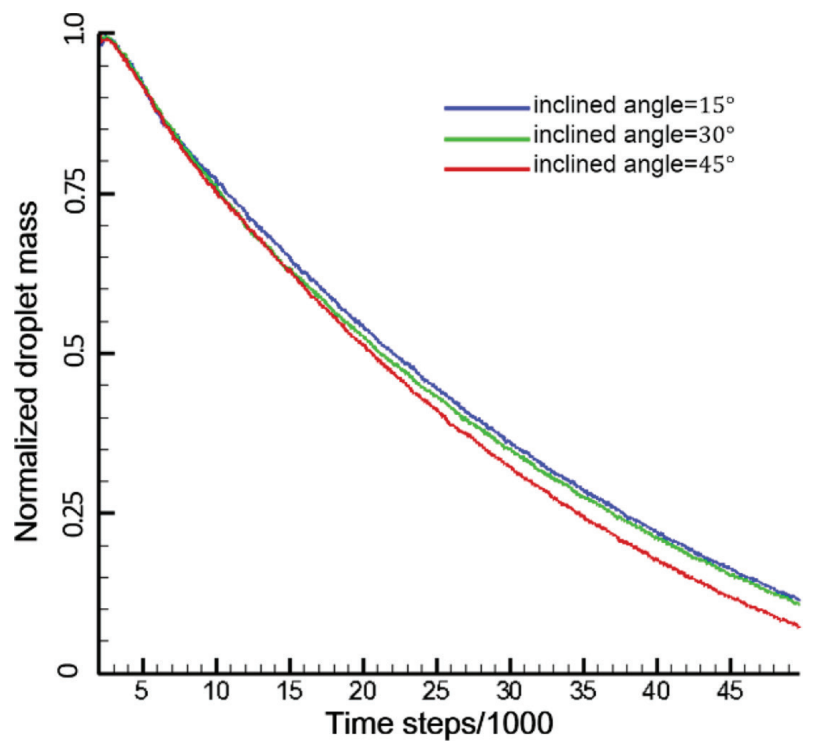

Figure 5: The mass of droplets versus time at different inclination angles.

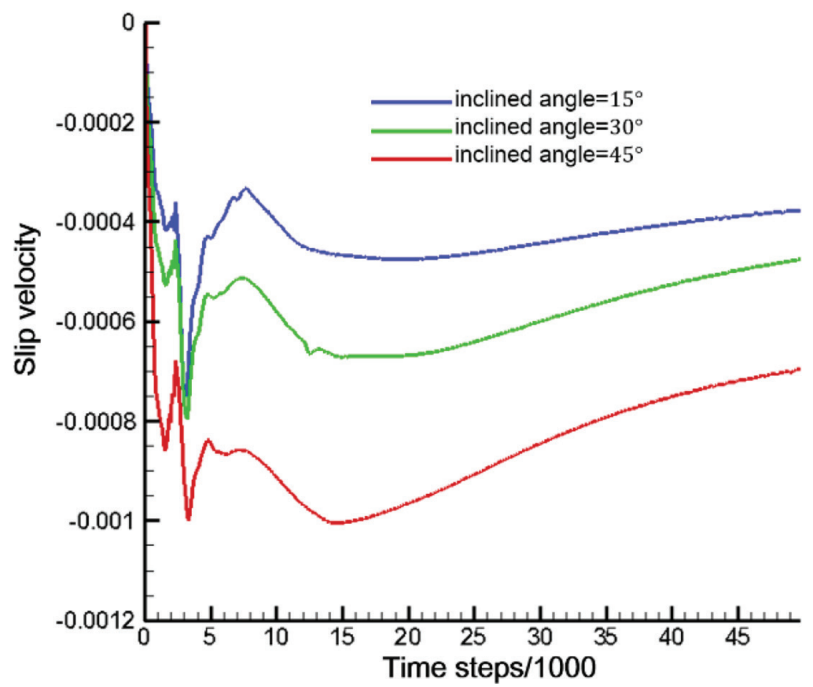

Figure 6: The slip velocity of droplets versus time at different inclination angles.

\section{CONCLUSIONS}

In this paper, the transient problem of $2 \mathrm{D}$ droplet deformation and evaporation was simulated by lattice Boltzmann model. This model consists of two parts: a multiphase LBM model and an energy equation model. The pseudo-potential model for multi-phase flows was used and the coexistence curve for P-R EOS was tested. A single droplet was placed in a gravitational field. The impingement and evaporation processes of this droplet were investigated. The results show that for a fixed initial velocity, when the inclined angel increases, droplet 
deformation is significant as the motion between droplet and the surface is strengthened and the droplet evaporation rate gets faster since the heat transfer is enhanced.

\section{ACKNOWLEDGEMENTS}

The work was sponsored by the National Natural Science Foundation of China (51276030), the Equipment Servicing Foundation of DUT (SYSWX201625) and the Fundamental Research Funds for the Central Universities (No. DUT16QY03, and DUT14ZD (G) 02).

\section{REFERENCES}

[1] Rein, M., Phenomena of liquid drop impact on solid and liquid surfaces. Fluid Dynamics Research, 12, pp. 61-93, 1993. https://doi.org/10.1016/0169-5983(93)90106-k

[2] Crafton, E.F. \& Black, W.Z., Heat transfer and evaporation rates of small liquid droplets on heated horizontal surfaces. International Journal of Heat and Mass Transfer, 47, pp. 1187-1200, 2004.

https://doi.org/10.1016/j.ijheatmasstransfer.2003.09.006

[3] Bhardwaj, R., Longtin, J.P. \& Attinger, D., Interfacial temperature measurements, highspeed visualization and finite-element simulations of droplet impact and evaporation on a solid surface. International Journal of Heat and Mass Transfer, 53, pp. 3733-3744, 2010. https://doi.org/10.1016/j.ijheatmasstransfer.2010.04.024

[4] Gunstensen, A.K., Rothman, D.H., Zaleski, S. \& Zanetti, G., Lattice Boltzmann model of immiscible fluids. Physical Review A, 43, pp. 4320-4327, 1991.

https://doi.org/10.1103/physreva.43.4320

[5] Shan, X. \& Chen, H., Simulation of non-ideal gases and liquid-gas phase transitions by lattice Boltzmann equation. Physical Review E, 47, pp. 1815-1819, 1994. https://doi.org/10.1103/physreve.47.1815

[6] Swift, M. R., Orlandini, E., Osborn, W. R. \& Yeomans, J. M., Lattice Boltzmann simulations of liquid-gas and binary fluid systems. Physical Review E, 54(5), pp. 5041-5052, 1996. https://doi.org/10.1103/physreve.54.5041

[7] He, X., Shan, X. \& Doolen, G. D., Discrete Boltzmann equation model for nonideal gases. Physical Review E, 57, pp. R13-R16, 1998.

https://doi.org/10.1103/physreve.57.r13

[8] Chen, L., Kang, Q., Mu, Y., He, Y.-L. \& Tao, W.-Q., A critical review of the pseudopotential multiphase lattice Boltzmann model: methods and applications. International Journal of Heat and Mass Transfer, 76, pp. 210-236, 2014. https://doi.org/10.1016/j.ijheatmasstransfer.2014.04.032

[9] Kupershtokh, A. L., Medvedev, D. A. \& Karpov, D. I., On equations of state in a lattice Boltzmann method, Computers \& Mathematics with Applications, 58, pp. 965-974, 2009. https://doi.org/10.1016/j.camwa.2009.02.024

[10] Gong, S. \& Cheng, P., Lattice Boltzmann simulation of periodic bubble nucleation, growth and departure from a heated surface in pool boiling. International Journal of Heat and Mass Transfer, 64, pp. 122-132, 2013. https://doi.org/10.1016/j.ijheatmasstransfer.2013.03.058

[11] Zarghami, A., Looije, N. \& Van den Akker, H., Assessment of interaction potential in simulating nonisothermal multiphase systems by means of lattice Boltzmann modeling. Physical Review E, 92, pp. 023307, 2015. https://doi.org/10.1103/physreve.92.023307 